\title{
BMJ Open Emotional states and coping methods in nursing and non-nursing students responding to COVID-19: a cross- sectional study in China
}

\author{
Qunfang Miao, ${ }^{1}$ Lin Xie, ${ }^{1}$ Bingyu Xing, ${ }^{1}$ Xiaolei Wang, ${ }^{1}$ Shiming Tang, ${ }^{2}$ \\ Hong Luo (1) ${ }^{3}$
}

To cite: Miao Q, Xie L, Xing B, et al. Emotional states and coping methods in nursing and non-nursing students responding to COVID-19: a cross-sectional study in China. BMJ Open 2021;11:e054007. doi:10.1136/ bmjopen-2021-054007

- Prepublication history for this paper is available online. To view these files, please visit the journal online (http://dx.doi. org/10.1136/bmjopen-2021054007).

Received 02 June 2021 Accepted 09 August 2021

Check for updates

(c) Author(s) (or their employer(s)) 2021. Re-use permitted under CC BY-NC. No commercial re-use. See rights and permissions. Published by BMJ.

${ }^{1}$ School of Medicine, Hangzhou Normal University, Hangzhou,

Zhejiang, China

${ }^{2}$ School of Education, Hangzhou Normal University, Hangzhou,

Zhengjiang, China

${ }^{3}$ Clinical Psychology, Hangzhou Normal University Affiliated Hospital, Hangzhou, China

Correspondence to

Dr Hong Luo;

m18106566750@163.com

\section{ABSTRACT}

Objectives To evaluate the emotion, coping strategy, dealing methods and their correlation in the COVID-19 outbreak among nursing and non-nursing students. Design and setting A cross-sectional online survey. Participants Full-time nursing and non-nursing undergraduate students.

Main outcome measure The Generalized Anxiety Disorder 7 (GAD-7) and Patient Health Questionnaire-9 (PHQ-9) were used to determine the emotional status in the COVID-19 pandemic among nursing and non-nursing students. Emotion Regulation Questionnaire (ERQ) was used to measure the emotion regulation strategies and the Simplified Coping Style Questionnaire (SCSQ) was used to evaluate the coping methods among nursing and nonnursing students.

Results In total, 746 students including 366 nursing students and 380 non-nursing students participated in the survey. Compared with the non-nursing students, a significant decrease was noticed in GAD-7 score $(p<0.01)$ and PHQ-9 $(p<0.01)$ in the nursing students. The cognition re-evaluation score in the nursing students was significantly lower than that of the non-nursing students $(p<0.05)$. In the nursing students, the score of anxiety was positively correlated with ERQ expression inhibition $(p<0.01)$ and SCSQ negative coping $(p<0.01)$, while the score of depression was also positively correlated with ERQ expression inhibition $(p<0.01)$ and SCSQ negative coping $(p<0.01)$. There was a negative correlation between $\operatorname{SCS} Q$ and the scores of anxiety $(p<0.05)$ and depression $(p<0.05)$. In the non-nursing students, the anxiety score was positively correlated with the SCSQ negative coping $(p<0.01)$, while the depression score was positively correlated with the ERQ expression inhibition $(p<0.01)$ and SCSQ negative coping $(p<0.01)$.

Conclusions COVID-19 affected the emotional status of nursing and non-nursing students. The emotional status was correlated with the emotional regulation and coping methods. Staff involved in the nursing professionals should pay attention to the psychological status of the nursing and non-nursing students, and give moderate psychological interference in the presence of COVID-19.

\section{INTRODUCTION}

COVID-19 is a new type of acute respiratory syndrome that emerged in Wuhan City, Hubei
Strengths and limitations of this study

- This survey was performed at the early stage of COVID-19 pandemic, and it is really a challenge for the questionnaire collection.

- The number of male nursing students was lower, which may lead to bias in the demographic information.

- We can further illustrate the stress pathway of nursing students in cases of severe public health events and then establish a model for the psychological health service.

- The data obtained in the setting of COVID-19 could be applied in the specific conditions cautiously.

Province in China in late December 2019. On 30 January 2020, COVID-19 was listed as the Public Health Emergency of International Concern by WHO. ${ }^{1}$ It has caused the outbreak of acute infectious pneumonia as it is highly epidemic, spreading to southern Asia (India, Nepal and Sri Lanka), eastern Asia (Japan and Korea), southeast Asia (Thailand, Singapore, Malaysia, Vietnam, Philippines and Cambodia), western Asia (United Arab Emirates), Europe (Germany, France, Italy, UK, Russia, Finland, Spain and Sweden), North America (USA and Canada) and Australia. ${ }^{2}{ }^{3}$ According to the survey, about $13 \%$ of the patients with confirmed 2019-novel coronavirus (nCoV) infection were reported to present severe respiratory symptoms, among which $2 \%$ died. $^{4}$ In a recent study, patients with COVID-19 usually present severe psychological stress, anxiety and depression. ${ }^{5}$ Gupta et al proposed a novel emotion care scheme to analyse multimodal textual data contained in the real-time tweets related to COVID-19. Besides, the authors investigated 8-scale emotions (ie, anger, anticipation, disgust, fear, joy, sadness, surprise and trust) induced by COVID-19. Adaptation 
reactions are helpful to the individuals when facing infectious diseases, which then contribute to the response to stress events. However, excessive emotional response may lead to somato-psychic disturbance in their daily lives.

In the presence of public health emergency, the doctors and nurses fulfilling the responsibility for helping the infected individuals by a certain disease are facing a huge physical and psychological stress. In a previous study that investigated the effects of SARS on the psychosocial behaviours in Toronto in 2003, Nickell et al focused on the respondents who were allied healthcare professionals, nurses and doctors serving as representatives of the hospital staff population as a whole. ${ }^{6}$ About $29 \%$ of the respondents reported SARS-related concern for their own or their families' health, while that rate among nurses was up to $45 \%$. According to the report by the National Health Commission of the People's Republic of China, more than 14000 doctors and nurses volunteered to manage the patients infected by the COVID-19 from all over China. ${ }^{7}$ They faced intensive treatment and nursing and bore a heavy occupational and psychological pressure. Up to now, a total of 1716 doctors and nurses were infected by COVID-19, accounting for about $3.8 \%$ of the total number of infected patients. In a recent survey on the psychological state of 207 nurses working in the COVID-19 designated hospital in Hangzhou, Zhang et $a l^{8}$ reported that most of the nurses showed psychological disorders. This should raise our attention on the psychological state of the staff involved in public health. COVID-19 could affect the emotion of the staff involving in medical and nursing professionals, and their response strategies would change under different emotions. ${ }^{9}$ The adults with anger and fear are more likely to respond positively to pressure events, while those with sorrow and depression had a greater tendency to choose a negative response. ${ }^{10}$ On this basis, more attention has been paid to the investigation on the relationship between individual response and psychological health.

Since the outbreak of COVID-19, more attention has been paid to the psychological health of nursing students. As the major reserve force of nursing professionals, nursing students are at a learning stage of their fields. Their inadequate understanding of COVID-19 and lack of clinical experiences would bring complex emotions. Although they have received professional nursing education and are well educated in the medical fields, they are indeed affected by emotions just like the non-nursing students. ${ }^{11}$ In colleges, nursing students are more likely to be trained on how to manage the infection, which is beneficial to the prevention of infectious diseases. ${ }^{12}$ Patel et al showed that a complete understanding on the cause, prevention and treatment of a certain disease would reduce the fear to the disease. Nevertheless, in a study investigating the psychological state of nursing students studying at a university located within the Wenchuan earthquake zone, it indicated that nursing students in areas affected by the Wenchuan earthquake suffer from anxiety and depression disorders. Participants most often employed basic problem-solving approaches to cope with psychological conflicts arising from their earthquake experience. ${ }^{13}$ In a cross-sectional study in Egypt, undergraduate university students were very prone to choose the problem-focused coping strategies during the COVID-19 pandemic. ${ }^{14}$ As COVID-19 is sudden onset with a rapid infection rate and a high mortality coexisting with unpredictability, little is known about the differences between nursing students and non-nursing counterparts in the emotional and coping strategies.

In this study, we aimed to investigate the emotion, coping strategy, dealing methods and their correlation in nursing and non-nursing students during the COVID-19 pandemic. In addition, we investigated the differences of the psychological status between nursing and non-nursing students, which contributed to the construction of psychological supporting system and coping strategies during the COVID-19 pandemic.

\section{MATERIALS AND METHODS}

\section{Patient and public involvement}

No patient involved.

\section{Subjects}

Each subject was well informed about the questionnaire and signed the informed consent online. In this study, we distributed our questionnaire to 770 students in a full-time university located in Hangzhou (Zhejiang Province, China) between 4 February and 10 February 2020. The questionnaire was distributed by online visiting to the undergraduate students in our university. All the subjects participated in the questionnaire voluntarily. For the quality control of the questionnaire, each online IP was only allowed to fill in one questionnaire. For the privacy of the subjects, their privacy information was not presented in it. On distribution of the questionnaire, automatic monitoring was performed to monitor the time duration each subject used on the questionnaire. Questionnaires accomplished in less than 180s or in a long time were excluded from the subsequent analysis. Before setting the items in the questionnaire, we needed to find out whether the subjects showed mental illness and COVID-19. In order to exclude the effects of the COVID-19 pandemic on psychological status, we excluded the subjects located in Wuhan City (Hubei Province, China). The inclusion criteria were as follows: (1) full-time undergraduate students; (2) those willing to participate in this survey; (3) those with no psychological illness or not infected by COVID19. Those with the following conditions were excluded from this study: (1) those with severe psychological problems or diseases; (2) infected by COVID-19; (3) those with an internet position at Wuhan City (Hubei, China).

\section{Questionnaire}

The questionnaire consisted of the following aspects: the generation information of the students, including gender, age and profession. 
Generalized Anxiety Disorder 7 (GAD-7) scale was used for the evaluation. A score range of 0-3 was set for each item, and the total score was 21 for the seven items. A score of 5, 10 and 15 was considered the threshold score for the slight, moderate and severe anxiety, respectively. In this study, the Chinese version of the GAD-7 scale was used. ${ }^{15} \mathrm{~A}$ total of 366 nursing students participated in the survey. The Cronbach $\alpha$ coefficient was 0.858 . In total, 380 non-nursing students participated in the survey, and the Cronbach $\alpha$ coefficient was 0.904 .

The Patient Health Questionnaire-9 (PHQ-9) consisted of nine items with a score range of 0-3 for each item (total score, 27). A higher score demonstrated a higher depression state. A score of 5, 10 and 15 was considered the threshold score for the slight, moderate and severe depression, respectively. The Chinese PHQ-9 version was adopted in this study. ${ }^{16}{ }^{17}$ In total, 366 nursing students participated in the survey, and the Cronbach $\alpha$ coefficient was 0.880 . The Cronbach $\alpha$ coefficient was 0.894 in the 380 non-nursing students.

Emotion Regulation Questionnaire (ERQ) consisted of 10 items involving two rating scales. The Chinese version of ERQ was adopted. ${ }^{18}$ Among the 366 nursing students, the Cronbach $\alpha$ of the two dimensions of the ERQ scale was 0.849 and 0.764 , respectively. Among the 388 nonnursing students, the Cronbach $\alpha$ of the two dimensions of the ERQ scale was 0.890 and 0.812 .

Simplified Coping Style Questionnaire (SCSQ) initially proposed by Xie in 1998 consisted of 20 items. Positive and negative coping was available in the Chinese version of SCSQ. ${ }^{19}$ The Cronbach $\alpha$ of the two dimensions of the SCSQ scale was 0.880 and 0.764 in the 366 nursing students, and 0.886 and 0.739 in the non-nursing students, respectively.

\section{Statistical analysis}

SPSS Statistics V.22.0 was used for the data analysis on collection of the questionnaire. All the measurement data were presented as mean $\pm \mathrm{SE}$. Independent sample t-test was used for the comparison of the GAD-7, PHQ-9, ERQ and SCSQ score between the nursing and non-nursing students. The gender difference between the nonnursing students was compared using the independent sample t-test. Pearson correlation analysis was conducted to analyse the correlation between anxiety, depression, regulatory strategy and coping methods. A p $<0.05$ was considered to be statistically significant.

\section{RESULTS}

\section{Demographics and description of quarantined persons}

In total, we distributed 770 questionnaires, and 746 (96.9\%) were finally collected, including 366 questionnaires collecting from nursing students and 380 from non-nursing students. Among the 366 nursing students, there were 20 men (5.5\%) and 346 women (94.5\%). The mean age for the respondents in the nursing students was $22.4 \pm 1.5$ years. Among the 380 non-nursing students, there were 93 men $(24.5 \%)$ and 287 women (75.5\%). The mean age was $22.1 \pm 1.2$ years.

The GAD-7 score for the students in the nursing profession was 2.12 \pm 2.63 . The PHQ-9 score was $3.34 \pm 4.13$. The ERQscore and SCSQ score was $43.45 \pm 6.61$ and $30.39 \pm 8.96$, respectively. For the non-nursing students, the GAD-7 score was 2.65 \pm 3.48 , and the PHQ-9 score was 3.74 \pm 4.79 . The ERQ score and SCSQ score was 44.10 \pm 7.59 and $31.00 \pm 9.41$, respectively. Student's t-test indicated that the number of nursing students with a mean GAD-7 score and PHQ-9 score of 5 or more was significantly lower than those of the non-nursing students $\left(t_{1}=-2.101\right.$, $\left.\mathrm{p}<0.01 ; \mathrm{t}_{2}=-1.372, \mathrm{p}<0.01\right)$. Moreover, no statistical differences were noticed in the GAD-7 score and PHQ-9 score in the nursing students and non-nursing students with mild, moderate and severe anxiety or depression, respectively (table 1, p>0.05). Student's t-test revealed the cognition re-evaluation score in the nursing students was significantly lower than that of the non-nursing students $(\mathrm{t}=-0.117, \mathrm{p}<0.05)$. For the comparison of SCSQ between the non-nursing students and nursing students, no statistical differences were noticed between them $(p>0.05$, table 2).

\section{Difference of non-nursing profession students of different genders in each scale}

For the non-nursing profession students, the score of anxiety and recognition re-evaluation of the male students was significantly lower than that of the female counterparts (anxiety: $\mathrm{t}_{1}=-3.494 ; \mathrm{p}<0.01$; recognition re-evaluation: $\left.\mathrm{t}_{2}=-1.250, \mathrm{p}<0.05\right)$. The expression inhibition in the male students was significantly higher than that of the female counterparts $(\mathrm{t}=3.569, \mathrm{p}<0.05$, table 3$)$. As the proportion of male students in the nursing profession was very small, there was no correlation analysis before the nursing profession students.

\section{Correlation between emotion, regulatory strategy and coping style}

Pearson regression analysis indicated a positive correlation between anxiety and depression of the nursing profession students and the ERQ expression inhibition and SCSQ negative coping $(\mathrm{p}<0.01)$. Meanwhile, there was a negative correlation between anxiety and depression of the nursing profession students and the positive coping of SCSQ $(p<0.05)$. For the non-nursing profession students, the depression was positively correlated with the expression inhibition of ERQ $(\mathrm{p}<0.01)$. In addition, anxiety and depression were positively correlated with the negative coping style of the SCSQ $(p<0.01$, table 4$)$.

\section{DISCUSSION}

It has been well acknowledged that there is a significant increase in the anxiety and depression among nursing or non-nursing students when facing severe stress events such as SARS and COVID-19. ${ }^{20}$ Anxiety is the most common emotional stress. Compared with anxiety, depression is 
Table 1 Detection rates of anxiety and depression of different levels and comparisons of Generalized Anxiety Disorder 7 (GAD-7) and Patient Health Questionnaire-9 (PHQ-9) scores between the nursing students and non-nursing counterparts

\begin{tabular}{|c|c|c|c|c|c|c|}
\hline & \multicolumn{2}{|c|}{ Nursing students ( $n=366$ ) } & \multicolumn{2}{|c|}{ Non-nursing students $(n=380)$} & \multirow[b]{2}{*}{$T / z$ value } & \multirow[b]{2}{*}{$P$ value } \\
\hline & Rate & Score & Rate & Score & & \\
\hline \multicolumn{7}{|c|}{ Anxiety according to GAD-7 } \\
\hline No anxiety & $84.7 \%$ & $1.21 \pm 1.28$ & $81.1 \%$ & $1.30 \pm 1.28$ & -0.864 & 0.472 \\
\hline Mild anxiety & $12.8 \%$ & $6.26 \pm 1.17$ & $13.4 \%$ & $6.24 \pm 1.18$ & 0.084 & 0.926 \\
\hline Moderate anxiety & $2.2 \%$ & $11.50 \pm 1.51$ & $3.4 \%$ & $11.69 \pm 1.70$ & -0.270 & 0.388 \\
\hline Severe anxiety & $0.3 \%$ & $15.00 \pm 0.00$ & $2.1 \%$ & $17.13 \pm 1.96$ & -1.192 & 0.444 \\
\hline Anxiety overall & $56(15.3 \%)$ & $7.16 \pm 2.45$ & $72(18.9 \%)$ & $8.43 \pm 3.97$ & -2.101 & $<0.001$ \\
\hline \multicolumn{7}{|c|}{ Depression according to PHQ-9 } \\
\hline No depression & $72.4 \%$ & $1.25 \pm 1.40$ & $70.8 \%$ & $1.30 \pm 1.35$ & -0.408 & 0.423 \\
\hline Mild depression & $18.8 \%$ & $6.74 \pm 1.48$ & $17.9 \%$ & $6.40 \pm 1.28$ & 1.445 & 0.119 \\
\hline Moderate depression & $6.6 \%$ & $11.50 \pm 1.22$ & $5.8 \%$ & $11.82 \pm 1.30$ & -1.009 & 0.888 \\
\hline Severe depression & $2.2 \%$ & $18.88 \pm 2.23$ & $5.5 \%$ & $18.05 \pm 3.57$ & -0.857 & 0.809 \\
\hline Depression overall & $101(27.6 \%)$ & $8.83 \pm 3.87$ & $111(29.2 \%)$ & $9.68 \pm 4.96$ & -1.372 & 0.003 \\
\hline
\end{tabular}

more likely to induce social dysfunction. In this study, the incidence of anxiety and depression among the nursing profession students was $15.3 \%$ and $27.6 \%$, which were higher than the incidence of the counterparts at normal conditions. In a previous study, Feng et al indicated the detection rate of anxiety and depression based on the SAS and SDS scale was $12.94 \%$ and $19.53 \%$, respectively. ${ }^{21}$ Meanwhile, in another study, the incidence of depression in the nursing students was $21.22 \% .^{22}$ In this study, the proportion of non-nursing students with anxiety and depression was $18.9 \%$ and $29.2 \%$, which was significantly higher than the rate of anxiety $(15.3 \%)$ and depression $(27.6 \%)$ in the nursing students. These indicated that severe stress events cause great threats to the psychological state of the nursing and non-nursing students, respectively. In this study, $2.5 \%$ of the nursing students showed moderate and severe anxiety, while $6.9 \%$ showed moderate and severe depression. For the non-nursing students, $6.9 \%$ showed moderate and severe anxiety, and $7.4 \%$ showed moderate and severe depression. For these students, further evaluation was required, together with drugs and psychological interference. Particularly, there were some students who presented thoughts of suicide, and immediate interferences were required. The campus is recommended to educate the students to adopt appropriate emotional regulation strategy and coping methods in the presence of public crisis. Besides, regular education on the COVID-19-related information including the onset, progression, outcome and prevention. The students with mild and moderate anxiety and depression should be educated to adapt to the anxiety and depression, together with positive communication with others and appropriate catharsis. Moreover, the students are recommended to obtain support from the hotline of the psychological stress, psychological counselling and training. Those with severe anxiety and depression are recommended to receive treatment in hospitals.

Interestingly, unlike the non-stress condition, the anxiety and depression score and incidence in the students of the nursing profession were significantly lower than that of the non-nursing students. The decline in the incidence and depression score may be related to the solid background of the nursing professions, which allows them a better understanding on the infectious disease. Meanwhile, these students were likely to receive education on the emotional management in campus, which contributed to their adaptation reaction of the emotion in the presence of COVID-19. This indeed is a positive feedback to the regulation of mental sources. ${ }^{12}$

The score of recognition re-evaluation in the nursing and non-nursing students was much higher than the expression inhibition score. Besides, the recognition re-evaluation score in these students was similar, which indicated that the undergraduate students showed a higher education level and a positive psychological state. These contributed to the generation of a well adaptation

Table 2 Comparison of Emotion Regulation Questionnaire (ERQ) and Simplified Coping Style Questionnaire (SCSQ)

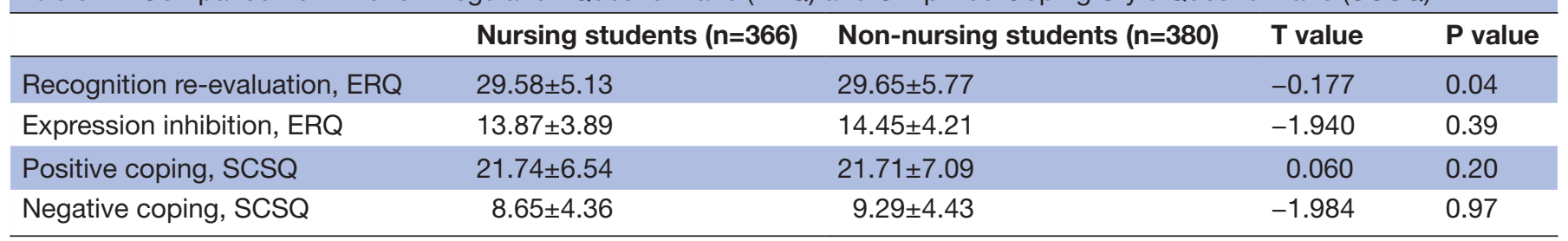


Table 3 Comparison of scale in different genders among the non-nursing students

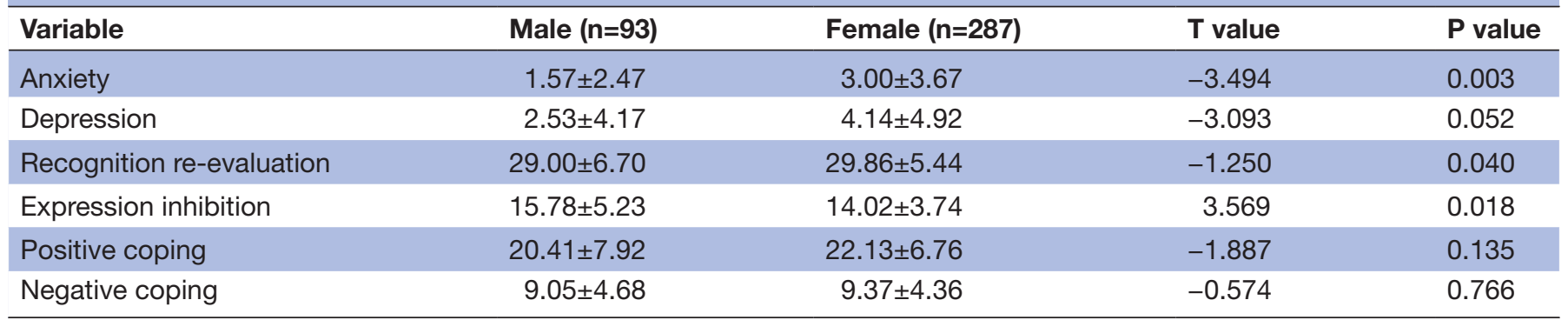

capacity to the COVID-19. As is known to all, a positive strategy for the recognition re-evaluation and a positive attitude towards stress such as frequent hand-washing and wearing a mask are helpful to keep a healthy state. Re-evaluation on the stress situation such as quarantine at home would promote the family relationship and attempt to recover the negative emotion. On this basis, anxiety was considered as a normal response to the crisis. In a previous study, Gross considered that emotional regulation was an essential part for the internal psychological capital, which meant the regulation involving multiple experiences, behaviours and psychological system. In addition, it was considered a functional reaction to the generation time, experience and expressional emotion. ${ }^{23}$ Individuals with recognition re-evaluation had a greater tendency to share their emotion, which obtained more social support. ${ }^{24}$ Despite the fact that quarantine at home limited the face-to-face communication to some extent, the online communication contributed to the students with a high recognition re-evaluation as they could obtain social support through communication via online system and/or telephone, which could attenuate the psychological pressure.

The mean score for the SCSQ positive coping dimension in the nursing students was similar to that of the non-nursing students, while the mean score for the negative coping dimension was lower than that of the nonnursing students. When facing the COVID-19, positive coping included wearing a mask, frequent handwashing and quarantine at home, together with obtaining social support. These measures were highly associated with the effective management of the Chinese government to face COVID-19. ${ }^{25}$ Since the COVID-19 pandemic, the Chinese government acts rapidly and adopts immediate and effective measures for the prevention and treatment to the civilians. In addition, the local authorities propose accurate and effective education to the civilians about COVID-19, and the disease is under control in time. ${ }^{26}$ Rare individuals showed negative coping and their patterns were presented in an individual pattern, such as their coping methods for depression, escape and selfblame. It is necessary to mention that there is no definite boundary between positive coping and negative coping when facing a severe public infection. Specially, there is indeed a relation between positive coping and negative coping. For the individuals placed in quarantine at home, their coping behaviours are still limited.

The uncontrollability and uncertainty of COVID-19 would lead to an increase in powerlessness and feeling of helplessness. Compared with the other conditions involving the utilisation of positive coping to the specific issues, the supporting means from the emotional aspects may provide more benefits to attenuate the pressure for the individuals placed in quarantine at home. Selfcomforting and accepting the reality were not effective ways for positive coping, but it was an effective coping strategy for COVID-19.

There might be interactions between emotional state and regulatory strategy. ${ }^{27}$ The emotional state would affect the selection of the regulatory strategy, while the regulatory strategy would modulate the emotional state. In a previous study, the individual responses to the emotional onset, experience and expression would play important roles in physical and mental health. Nursing students using positive recognition re-evaluation strategy were more positive in their daily lives to the normal affairs, and they were apt to alternate their emotional state based on their knowledge to the emotional stress. This contributed to the attenuation of negative emotion, which would prevent the individuals from experiencing

Table 4 Correlation between anxiety, depression and regulatory strategy and coping methods

\begin{tabular}{|c|c|c|c|c|c|c|c|c|}
\hline \multirow[b]{2}{*}{ Variable } & \multicolumn{4}{|c|}{ Nursing students $(n=366)$} & \multicolumn{4}{|c|}{ Non-nursing students $(n=380)$} \\
\hline & $\begin{array}{l}\text { Recognition } \\
\text { re-evaluation }\end{array}$ & $\begin{array}{l}\text { Expression } \\
\text { inhibition }\end{array}$ & $\begin{array}{l}\text { Positive } \\
\text { coping }\end{array}$ & $\begin{array}{l}\text { Negative } \\
\text { coping }\end{array}$ & $\begin{array}{l}\text { Recognition } \\
\text { re-evaluation }\end{array}$ & $\begin{array}{l}\text { Expression } \\
\text { inhibition }\end{array}$ & $\begin{array}{l}\text { Positive } \\
\text { coping }\end{array}$ & $\begin{array}{l}\text { Negative } \\
\text { coping }\end{array}$ \\
\hline PHQ-9 & -0.035 & $0.192^{\star \star}$ & $-0.190^{\star *}$ & $0.176^{\star *}$ & -0.065 & $0.145^{\star \star}$ & -0.077 & $0.248^{\star \star}$ \\
\hline
\end{tabular}

${ }^{*} \mathrm{p}<0.05 ;{ }^{* *} \mathrm{p}<0.01$.

GAD-7, Generalized Anxiety Disorder 7; PHQ-9, Patient Health Questionnaire-9. 
depression and anxiety. In this study, there was a positive correlation between depression emotion and expression inhibition of ERQ in the nursing and non-nursing students $(p<0.01)$, especially the nursing students. Those with obvious depression emotion were mainly featured by sorrow and loneliness. They did not tend to express their feelings in cases of negative emotion, and they would emerge in it. When evoked by anxiety and nervousness, the individuals willing to express their depression would attempt to suppress the emotion. This would lead to negative effects to themselves and experience on the negative feeling, which was harmful to the individuals. The students not willing to express their emotion under a high pressure were not likely to acknowledge their anxiety and depression. They considered this as immoderate and would not be accepted by the others even if they expressed their feeling.

In a previous study, $\operatorname{Ray}^{28}$ proposed a risk of up to $43.3 \%$ for the psychological issues for the individuals lacking effective coping methods in the presence of high stress, which was about twofold higher than that of the normal counterparts. Appropriate coping was effective for the management and attenuation of the over-response and unhealthy psychological behaviours, which would alternate the subjective knowledge of an individual. Subsequently, it would relieve the psychological nervousness and maintain the psychological balance. In contrast, negative coping would lead to anxiety. In our survey, coping strategy was correlated with anxiety. Besides, there was a positive correlation between anxiety, depression and negative coping. Similar with the previous study, ${ }^{29}$ those with high anxiety were more likely to select a negative coping method, while those with lower anxiety were more likely to select a positive coping method. This indicated that staff involved in the nursing education should pay attention to the psychological status of the nursing students and propose appropriate psychological interference, and integrate the systemic training on the outbreak of public health events to the nursing education. For the staff involved in the education of non-nursing students, attention should be paid to the psychological health of the students in cases of emergency events, and improve the psychological health through regulating the emotion and coping strategies. In addition, the non-nursing students should be educated about the emergency events, together with the prevention of the disease. In addition, measures should be taken to protect the students from emergency events that may hamper the psychological health in order to improve the psychological health through regulating the emotional regulation and coping methods.

In this survey, the male nursing students accounted for about $5.5 \%$, and then no differential analysis was conducted in views of gender. For the non-nursing students, Student's t-test indicated statistical differences in the emotional states and regulatory strategy between the male and female counterparts. The female students were more sensitive in feeling and emotional experience than the male students, which would easily bring in negative effects in the presence of COVID-19. This indicated that the female student had a greater tendency to use the emotion regulation than the male student; however, it triggered no benefits to prevent the development of emotional problems. Instead, the female students were more likely to adopt maladjusted strategies, which may lead to more emotional disorders easily. ${ }^{30}$ Meanwhile, in a meta-analysis, although there were small differences in gender, there were large variations in the emotional expression. Compared with men, women tended to express more positive feeling and negative feeling (eg, sorrow and anxiety). ${ }^{31}$ This was in line with the previous study, in which the female student was more likely to bear a larger stress and generate more psychological nervousness on seeing any information about SARS such as infection rate, death and other online information. On this basis, the male student was relatively lonely in the presence of COVID-19 even if they were not infected. They would not communicate with others and chose negative coping methods (eg, avoiding seeing or self-blame), which resulted in inadequate social and emotional support.

\section{LIMITATIONS}

This survey was performed during the epidemic stage of COVID-19, and it is really a problem for the sample collection. Thus, convenient sampling was used, which hampered the representativeness of the samples. Meanwhile, the online questionnaire would be affected by the social network, which may lead to generation of bias in the data collection. Furthermore, the number of male students in the nursing profession was lower, which may lead to bias in the demographic information. Indeed, there are really some aspects to be improved. We can further illustrate the stress pathway of the nursing and non-nursing students in cases of severe public health events, and then establish a model for the psychological health service. The data obtained in the setting of COVID-19 could be applied in the specific conditions cautiously.

\section{CONCLUSION}

COVID-19 affected the emotional status of nursing and non-nursing students. The emotional status of the nursing students was less likely to be affected by COVID-19 compared with the non-nursing counterparts. The emotional status was closely related to the emotion regulation and coping methods. Therefore, more attention should be paid to the psychological status of the nursing and non-nursing students, together with appropriate psychological interference in the presence of COVID-19.

Contributors LX and BX collected the questionnaire information; XW analysed the data; ST searched and reviewed the literature; QM wrote the manuscript and acted as guarantor for the overall content of the article; $\mathrm{HL}$ revised the manuscript.

Funding The authors have not declared a specific grant for this research from any funding agency in the public, commercial or not-for-profit sectors. 
Competing interests None declared.

Patient and public involvement Patients and/or the public were involved in the design, or conduct, or reporting, or dissemination plans of this research. Refer to the Methods section for further details.

Patient consent for publication Not required.

Ethics approval This study was approved by the Ethical Committee of Hangzhou Normal University (No. 20190066).

Provenance and peer review Not commissioned; externally peer reviewed.

Data availability statement Data are available on reasonable request.

Open access This is an open access article distributed in accordance with the Creative Commons Attribution Non Commercial (CC BY-NC 4.0) license, which permits others to distribute, remix, adapt, build upon this work non-commercially, and license their derivative works on different terms, provided the original work is properly cited, appropriate credit is given, any changes made indicated, and the use is non-commercial. See: http://creativecommons.org/licenses/by-nc/4.0/.

\section{ORCID iD}

Hong Luo http://orcid.org/0000-0002-0915-7149

\section{REFERENCES}

1 Bao Y, Sun Y, Meng S, et al. 2019-nCoV epidemic: address mental health care to empower society. Lancet 2020;395:e37-8.

2 Lai C-C, Shih T-P, Ko W-C, et al. Severe acute respiratory syndrome coronavirus 2 (SARS-CoV-2) and coronavirus disease-2019 (COVID-19): the epidemic and the challenges. Int J Antimicrob Agents 2020;55:105924.

3 Zu ZY, Jiang MD, Xu PP, et al. Coronavirus disease 2019 (COVID-19): a perspective from China. Radiology 2020;296:E15-25.

4 Hossain MM, Tasnim S, Sultana A, et al. Epidemiology of mental health problems in COVID-19: a review. F1000Res 2020;9:636.

5 Gupta V, Jain N, Katariya P, et al. An emotion care model using multimodal textual analysis on COVID-19. Chaos Solitons Fractals 2021;144:110708.

6 Nickell LA, Crighton EJ, Tracy CS, et al. Psychosocial effects of SARS on hospital staff: survey of a large tertiary care institution. CMAJ 2004;170:793-8.

7 Sun P, Lu X, Xu C, et al. Understanding of COVID-19 based on current evidence. J Med Virol 2020;92:548-51.

8 Zhang WH, Li E, Zhang Y. Investigation and countermeasures of anxiety of nurses in a designated hospital of novel coronavirus pneumonia in Hangzhou. Health Research 2020;40:130-3.

9 Charles ST, Reynolds CA, Gatz M. Age-related differences and change in positive and negative affect over 23 years. $J$ Pers Soc Psychol 2001;80:136-51.

10 Cai Z, Zheng S, Huang Y, et al. Emotional and cognitive responses and behavioral coping of Chinese medical workers and general population during the pandemic of COVID-19. Int J Environ Res Public Health 2020;17:6198

11 Albaqawi HM, Alquwez N, Balay-Odao E, et al. Nursing students' perceptions, knowledge, and preventive behaviors toward COVID-19: a multi-university study. Front Public Health 2020;8:573390.
12 Patel I, Guy J, Han Y, et al. Effects of Ebola virus disease education on student health professionals. Curr Pharm Teach Learn 2018;10:651-6.

13 Chen $\mathrm{H}$, Feng L, Shen W-W, et al. [A study on the psychological state of nurse-students of a university in affected areas of Wenchuan earthquake]. Hu Li Za Zhi 2009;56:30-8.

14 El-Monshed AH, El-Adl AA, Ali AS, et al. University students under lockdown, the psychosocial effects and coping strategies during COVID-19 pandemic: a cross sectional study in Egypt. J Am Coll Health 2021;2:1-12.

15 X Y H, Li C, Qian J. Reliability and validity of a generalized anxiety scale in general hospital outpatients. Shanghai Archives of Psychiatry 2010;22:200-3.

16 Kroenke K, Spitzer RL, Williams JB. The PHQ-9: validity of a brief depression severity measure. J Gen Intern Med 2001;16:606-13.

17 Bian C, He X, Qian J. The reliability and validity of a modified patient health questionnaire for screening depressive syndrome in general hospital outpatients. Journal of Tongji University (Medical Science) 2009;5.

18 Wang L, Liu H C LZQ, et al. A study on the validity of the Chinese version of emotional regulation questionnaire. Chinese Journal of Health Psychology 2007;15:503-5.

19 Xie YN. A preliminary study on the reliability and validity of the simple coping style scale. Chinese Journal of Clinical Psychology 1998;02:53-4.

20 Smith GD, Ng F, Ho Cheung Li W. COVID-19: emerging compassion, courage and resilience in the face of misinformation and adversity. $J$ Clin Nurs 2020;29:1425-8.

21 Feng FL, Wang CY, Wang YR. Investigation and analysis of anxiety and depression in medical college students. Journal of Hebei Medical University 2018;44:39:636-9.

22 Zhu GY. Analysis on the results of the investigation on depression and emotional status of higher nursing students. China Maternal and Child Health 2007;25:3546-7.

23 Gross JJ. Emotion regulation: past, present, future. Cogn Emot 1999:13:551-73.

24 F F H, Yang YY, Shi WD. Effects of college students'autistic traits on friendship quality: mediating role of emotion regulation strategies. Psychological Research 2019;12:556-63.

25 Huang G C ZX. Crisis management: effective measures for the COVID-19 treatment and prevention in campus. Liaoning Education 2020;49:15-18

26 Huang YZ, Xiao XW, Tao Y, et al. Prevention and stress reaction towards the COVID-19 in the students in campus. Zhong Guo Jian Kang Xin Li Xue Za Zhi 2020;28:1361-6.

27 Guo XQ, Wang L. Relationship between emotion regulation strategies and regulation patterns and anxiety among senior students. ] Chinese Journal of Mental Health 2016;30:706-11.

28 Ray C, Lindop J, Gibson S. The concept of coping. Psychol Med 1982;12:385-95.

29 Pan X, Kang J, S H L. A study on coping style and anxiety of medical college students. Chinese Medical Ethics 2011;24:547-8.

30 Sanchis-Sanchis A, Grau MD, Moliner A-R, et al. Effects of age and gender in emotion regulation of children and adolescents. Front Psychol 2020;11:946.

31 Chaplin TM, Aldao A. Gender differences in emotion expression in children: a meta-analytic review. Psychol Bull 2013;139:735-65. 